\title{
Function and regulation of tau conformations in the development and treatment of traumatic brain injury and neurodegeneration
}

Onder Albayram ${ }^{\dagger}$, Megan K. Herbert ${ }^{\dagger}$, Asami Kondo, Cheng-Yu Tsai, Sean Baxley, Xiaolan Lian, Madison Hansen, Xiao Zhen Zhou* and Kun Ping Lu*

\begin{abstract}
One of the two common hallmark lesions of Alzheimer's disease (AD) brains is neurofibrillary tangles (NFTs), which are composed of hyperphosphorylated tau protein ( $p$-tau). NFTs are also a defining feature of other neurodegenerative disorders and have recently been identified in the brains of patients suffering from chronic traumatic encephalopathy (CTE). However, NFTs are not normally observed in traumatic brain injury (TBI) until months or years after injury. This raises the question of whether NFTs are a cause or a consequence of long-term neurodegeneration following TBI. Two conformations of phosphorylated tau, cis p-tau and trans p-tau, which are regulated by the peptidyl-prolyl isomerase Pin1, have been previously identified. By generating a polyclonal and monoclonal antibody (Ab) pair capable of distinguishing between cis and trans isoforms of p-tau (cis p-tau and trans p-tau, respectively), cis p-tau was identified as a precursor of tau pathology and an early driver of neurodegeneration in AD, TBI and CTE. Histological studies shows the appearance of robust cis p-tau in the early stages of human mild cognitive impairment (MCI), AD and CTE brains, as well as after sport- and military-related TBI. Notably, cis p-tau appears within hours after closed head injury and long before other known pathogenic p-tau conformations including oligomers, pre-fibrillary tangles and NFTs. Importantly, cis p-tau monoclonal antibody treatment not only eliminates cis p-tau induction and tau pathology, but also restores many neuropathological and functional outcome in TBI mouse models. Thus, cis p-tau is an early driver of tau pathology in TBI and CTE and detection of cis p-tau in human bodily fluids could potentially provide new diagnostic and prognostic tools. Furthermore, humanization of the cis p-tau antibody could ultimately be developed as a new treatment for $\mathrm{AD}, \mathrm{TBI}$ and $\mathrm{CTE}$.
\end{abstract}

\section{Background}

Tau protein is a member of the microtubule-associated family of proteins which are expressed predominantly in the brain. Tau's primary functions, which include the stabilization of microtubules and the coordinated movement of molecules along the microtubule, are tightly regulated by phosphorylation [1-3]. In its native state, tau

\footnotetext{
*Correspondence: xzhou@bidmc.harvard.edu; klu@bidmc.harvard.edu ${ }^{\dagger}$ Onder Albayram and Megan K. Herbert contributed equally to this work Division of Translational Therapeutics, Department of Medicine and the Cancer Center, Beth Israel Deaconess Medical Center, Harvard Medical School, 330 Brookline Avenue, CLS 0408, Boston, MA, USA
}

is present in a stable, unfolded monomeric conformation. However, via as yet unknown mechanisms, tau becomes aberrantly phosphorylated, or hyperphosphorylated, and aggregated in several neurodegenerative diseases, collectively known as tauopathies $[2,3]$.

Although human tau is predominantly expressed in neurons, it can also be found in astrocytes and oligodendrocytes [4]. In the central nervous system, alternate splicing of exon 2, 3 and 10 leads to the generation of six isoforms of tau which range from 352 to 441 amino acids in length and $60-74 \mathrm{kDa}$ in weight as determined by SDS-PAGE analyses [5]. The variability in these isoforms derives from the presence or absence of exon inserts $(0$, 
1 or 2) in tau's $\mathrm{N}$-terminal region and the presence or absence of microtubule binding repeat domains in tau's C-terminal region [6]. Highly phosphorylated tau with a 3-repeat domain in the C-terminus predominates during early stages of development whereas a $\sim 1: 1$ ratio of 3-repeat:4-repeat tau is present in adults. Trace amounts of tau are also detectable in peripheral organs such as the heart, kidney, lungs, muscle, pancreas and testis but this peripheral tau is larger than brain tau with an additional $\mathrm{N}$-terminal sequence tau encoded by exon $4 \mathrm{~A}$. Thus, it is sometimes referred to as 'big tau' [6].

Phosphorylation of tau decreases normally with age and coincides with the development of phosphatases [7]. However, in tauopathies, the aberrant phosphorylation of tau leads to abnormal accumulations of tau in the brain [8]. The most archetypal tau aggregations occur in Alzheimer's disease (AD) in which hyperphosphorylated tau forms aggregates within the cell bodies known as neurofibrillary tangles (NFTs). In addition to AD there are several well-established neurodegenerative tauopathies, which include fronto-temporal dementia, Pick's disease, amyotrophic lateral sclerosis (ALS), progressive supranuclear palsy (PSP) and corticobasal dementia (CBD) as well as Parkinson's disease with dementia [9]. More recently, abnormal accumulations of tau have been associated with chronic traumatic encephalopathy (CTE) and traumatic brain injury (TBI), particularly in sportsrelated injuries exposing athletes to repeated mild traumatic brain injury (rmTBI), with or without concussion, and military personnel exposed to repeated explosive blast injuries [10-12].

\section{Post-translational modifications and tau aggregation in neurodegenerative diseases}

Tau can be post-translationally modified in several ways including phosphorylation, acetylation, glycation, prolylisomerization, cleavage or truncation, nitration, polyamination, ubiquitination, sumoylation, oxidation and aggregation [13-15]. The most well studied of these, and arguably one of the most important, is the phosphorylation of tau [16]. Tau in its dephosphorylated state is not prone to aggregation but phosphorylation of tau, which is required for regulation of its physiological functions, can increase or reduce its stability. Although debate continues regarding the contribution of tau to neurodegeneration, there is mounting evidence to suggest that the phosphorylation of tau, or more notably the hyperphosphorylation of tau, may lead to its increased aggregability, eventually leading to neuropathogenicity such as NFTs $[17,18]$. In AD, hyperphosphorylated tau in NFTs is composed of approximately equal amounts of both $3 R$ and $4 \mathrm{R}$ tau whereas other diseases are associated with a higher ratio of 3R:4R (Pick's disease) or a higher ratio of
4R:3R (CBD and PSP). The appearance and localisation of tau aggregates in different neurodegenerative diseases also varies widely $[19,20]$.

\section{Hyperphosphorylation of tau}

The primary mode of regulation of tau function occurs via phosphorylation at specific sites and coordinated phosphorylation events are necessary for proper neurite outgrowth and axonal transport processes [21]. While the exact role of abnormal phosphorylation of tau in tauopathies is unclear, there is substantial evidence to show that hyperphosphorylation plays a key role in the development of $\mathrm{AD}$ and a myriad of other neurodegenerative diseases associated with pathological tau [18, 22]. Hyperphosphorylation induces the formation of tau aggregates with highly phosphorylated tau showing self-assembly in vitro. Mutations at the Ser422 location lead to a significant increase in tau-tau propensity [17]. Pre-tangle formation in specific brain regions has been shown to hinder memory, disrupt synaptic function, and produce a rapid impairment of long-term potentiation (LTP). These toxic molecules can also catalyse neuronal loss and the accumulation of intracellular neurofibrillary tangles (NFTs) composed mainly of tau protein [17, 23]. Growing evidence also proposes that these potentially neurotoxic conglomerations perhaps act in a prion-like manner, but additional research will be required to elucidate their exact mechanism of action [24]. Interestingly, hyperphosphorylated tau aggregates reach a neurotoxic state prior to the formation of neurofibrillary tangles, thus making tau an enticing new target for preventative treatments of tau pathology [25]. Along with increased tau-tau affinity, hyperphosphorylation has also been correlated with a loss of normal tau physiological function. Neurons labeled with antibodies to recognize phosphoThr231 and phospho-Ser262 [26] display significantly decreased normal physiological interactions of tau with microtubules upon phosphorylation [25, 27] greatly diminishing the ability of this protein to bind to, as well as stabilize, microtubules. It is therefore suggested that the loss of normal physiological functions, as well as the onset of toxic characteristics driven by the aberrant phosphorylation of tau, likely contribute synergistically to tau pathology.

\section{Tau in traumatic brain injury and chronic traumatic encephalopathy}

TBI has been identified as a major risk factor for CTE and $\mathrm{AD}$, which are characterized by abnormally phosphorylated tau aggregates in neurons and glia of multiple brain regions $[28,29]$. For example, one in three NFL players are expected to experience cognitive problems in their lifetime. TBI is a leading cause of death or disability 
of children and young adults aged 1-44 and also affects one in five veterans of the Afghanistan and Iraq conflicts [29]. However, pathogenic mechanisms leading from acute TBI to chronic neurodegeneration are virtually unknown. There is no effective treatment available for mitigating secondary injury after acute TBI and preventing the late development of AD or CTE. Neurofibrillary tangles (NFTs) composed of phosphorylated tau are a neuropathological hallmark not only of AD but also CTE $[11,29]$. Furthermore, the tau isoform and hyperphosphorylation profiles of tau tangles purified from boxer CTE brains and AD brains are indistinguishable [30, 31]. Tau in these diseases is commonly hyperphosphorylated on Ser or Thr residues, especially those preceding a Pro residue (pSer/Thr-Pro) and such phosphorylation precedes tangle formation and neurodegeneration. However, it is not fully understood how phosphorylation causes tau, which serves a vital physiologic function in healthy neurons, to become pathogenic. Until recently, whether tau is further regulated after phosphorylation and if the pathogenic phosphorylated tau can be blocked without affecting physiological tau were not known.

In rodent models mimicking the effects of TBI, a notable increase in total tau levels and p-tau pretangle conformations, in conjunction with white matter degradation and increased neuroinflammation, have been found 2-3 months after post-injury [32]. These findings indicate a potential role for tau in the propagation of neuropathology in TBI and illustrate a possible bridge between TBI and CTE.

\section{The emergence of a new tau isoform-cis p-tau}

Proline-directed Ser/Thr phosphorylation is a central common signaling mechanism in the cell. We have found that certain pSer/Thr-Pro motifs exist in cis or trans conformations, and their conversion and function is further regulated by the unique prolyl isomerase Pin1 [33]. It has been shown that Pin1 is pivotal in protecting against age-dependent tau aggregation in $\mathrm{AD}$ by isomerizing the phosphorylated T231-P motif in tau (pT231-tau or p-tau) from cis to trans p-tau [33-35]. Furthermore, Pin1 is required for the regulated dephosphorylation of tau during microtubule stabilisation. Therefore, in the absence of Pin1, tau cannot be properly dephosphorylated and the ratio of cis p-tau to trans p-tau ratio is increased [33]. This discovery indicates that cis $\mathrm{p}$-tau may be pathologically important and targetable. Normally, more than $90 \%$ of conventional pT231-tau peptides are in trans p-tau conformation [36] and therefore can only be used to generate trans mAbs [36, 37], which would likely reduce physiological tau. Using novel peptide chemistry, we recently generated not only trans p-tau-specific but also cis p-tau-specific antibodies which we used to discover a previously unrecognized pathogenic cis p-tau which appears early-on in the disease process and which is not readily or dynamically converted to trans in vivo in the absence of Pin1 [37].

Deficits in the function of Pin1, correlating with an increase in cis- $\mathrm{p}$-tau have already been implicated in early $\mathrm{AD}[37,38]$. In particular, Pin1 down-regulation [33, 35], Cys113 oxidation [39] and phosphorylation at residue $\mathrm{S} 71[39,40]$ have been shown to contribute to the aberrant tau function in AD. Down-regulation of Pin1 via serum depletion or Cys113 oxidation via hypoxia in cultured neurons correlates well with increases in cis p-tau. Pin1 binds to tau primarily at the T231 residue catalysing the conversion from cis to trans p-tau [33, 41]. Since the phosphorylation of T231 is also an early step in tangle formation [42], Pin1 plays a critical role in reversing phosphorylation, preventing hyperphosphorylation and preventing accumulation of cis p-tau [33, 37, 38]. We further discovered that cis, but not trans, p-tau appears early in the neurons of human brains affected by MCI. We found that cis p-tau accumulates exclusively in the dystrophic neurites of degenerating neurons as $\mathrm{AD}$ progresses and correlates well with cognitive deficits [38]. Moreover, cis, but not trans, p-tau loses its normal microtubule-assembling ability, and gains toxic function, being resistant to dephosphorylation and degradation, and prone to aggregation [37, 38, 43]. Thus, cis p-tau may be a precursor of tau pathologies and an early driver of neurodegeneration which may have the potential for neuron-to-neuron transmission in TBI, CTE and AD.

Using cis and trans tau-specific monoclonal antibodies it has been possible to show that robust cis $\mathrm{p}$-tau appears early in human MCI, AD and CTE brains, as well as after sport- and military-related TBI and long before tau aggregates or fibrils can be detected [43]. In TBI closedhead injury mouse models, cis, but not trans, p-tau was readily induced at $12 \mathrm{~h}$ post-injury and further increased with time, localizing mainly to axons leading to axonopathy. This occurred long before commonly other known pathological tau, such as fibrillary tau and tau oligomers, could be detected. Furthermore, cis p-tau monoclonal antibody $(\mathrm{mAb})$ treatment eliminated cis $\mathrm{p}$-tau induction as well as cis p-tau-induced neurotoxicity in stressed neuron models in vitro and in TBI mouse models in vivo [43]. Of note, recent studies have shown the presence of tau oligomers within $48 \mathrm{~h}$ of severe open-head injury in mouse models [44], which were not observed in our closed-head injury model [43] (and unpublished results). Thus, neutralizing mAbs against the early and toxic cis p-tau reduce pathological tau without affecting healthy trans $\mathrm{p}$-tau, and have the potential to be highly efficacious and specific in halting or preventing tau pathology and memory loss in TBI, CTE and AD at early stages 
(Fig. 1). The mechanism by which TBI induces robust cis p-tau induction is currently unclear. However, it is likely that TBI, as in stroke and AD, causes the induction of the stress protein death-activated protein kinase 1 (DAPK1), which is responsible for the phosphorylation and inactivation of Pin1 [40]. Therefore, increases in DAPK1 will likely cause inactivation of Pin1 and subsequent induction of cis p-tau.

\section{Discovery of cistauosis and its role in early common disease mechanism in TBI, CTE and AD, which can be blocked by cis $\mathrm{mAb}$}

Immunotherapy against tau is attracting attention because active or passive immunization against p-tau tangle epitopes reduces the initial formation of pathological tau 'seeds' and subsequent spreading of tau aggregates. Although extensive tangles are a neuropathological signature in CTE in sport and military, evidence of tau pathology is not readily detectable acutely after TBI in humans and mouse models using other commonly known antibodies/techniques. However, we found robust cis p-tau in human CTE brains. Surprisingly, after TBI in mice and stress in vitro, neurons acutely produce cis $\mathrm{p}$-tau notable at axons, which disrupts axonal microtubules and axonal mitochondrial transport, spreads to other neurons, and leads to apoptosis [43]. This process, which we term 'cistauosis', appears long before other tau oligomers and fibrils but can be blocked by cis mAb and enhanced by trans mAb [43]. Treating TBI mice with cis mAb blocks early cistauosis, prevents the development and spread of tau aggregates such as oligomers and fibrils, and restores many TBI-related structural and functional

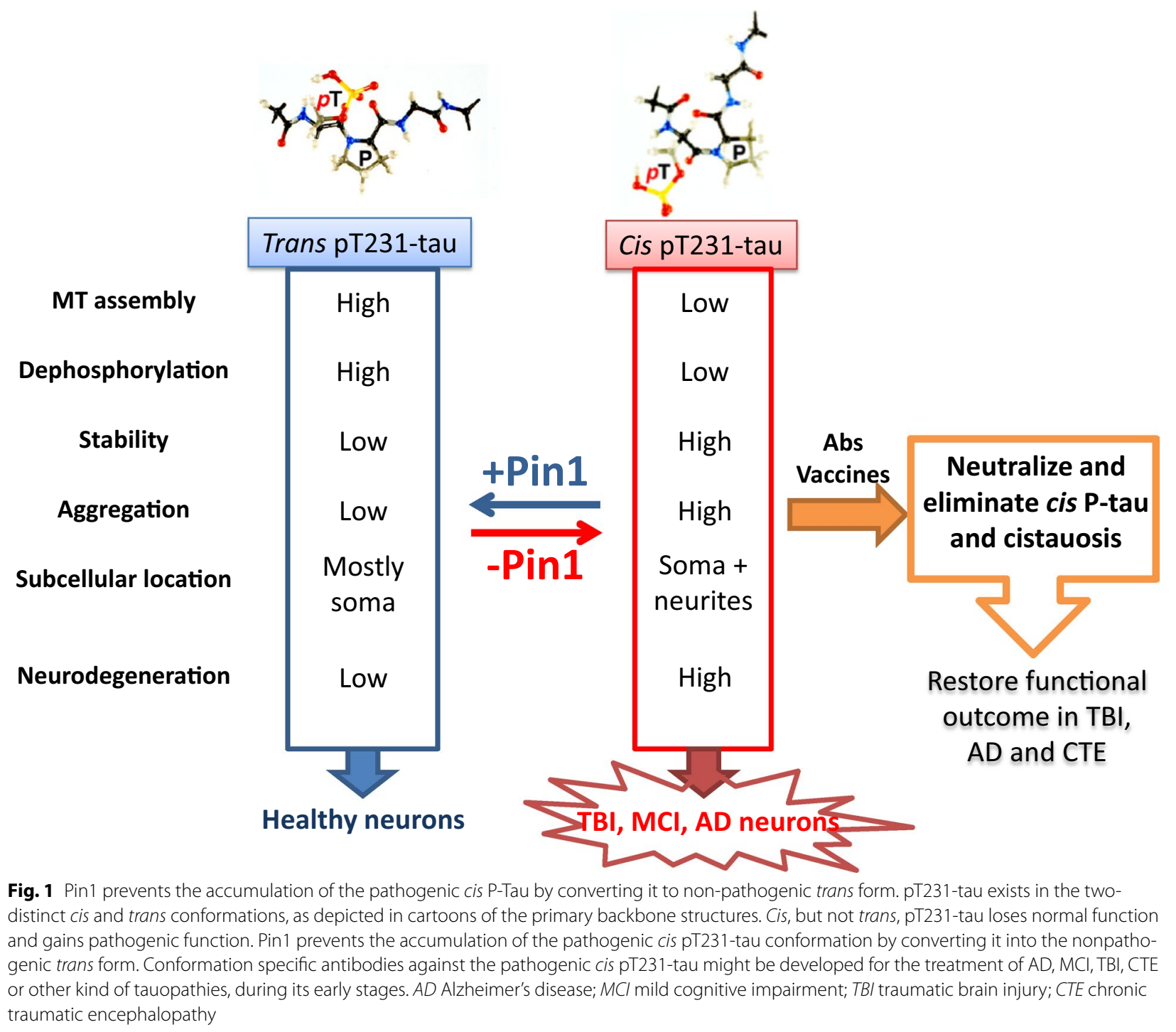


changes. Thus, cis, but not trans, p-tau is a likely pathogenic form of tau in $\mathrm{MCI}$ and $\mathrm{AD}$ and the development of humanised antibodies directed against pathogenic cis p-tau could be useful for the early diagnosis and treatment of AD, TBI and CTE.

\section{Treatments to reduce pathogenic tau isoforms in AD, TBI and CTE}

Currently there are no effective treatment options for patients with AD. Drug trials targeting reduction of the amyloid beta deposits have been shown to be efficacious in reducing amyloid beta deposits in the brain but there has been no convincing evidence that there is improvement in cognitive function [45]. The disappointing results from amyloid beta treatment studies has caused a shift in focus to the treatment of tau in AD. More recently, CSF biomarker studies have indicated that tau is superior to amyloid beta markers in following the progression of cognitive deficits in $\mathrm{AD}$ and other tauopathies [46, 47]. Furthermore, a newly developed PET technique to track changes in tau deposition in living patients with AD also shows that tau deposition more closely tracked dementia status and was a better indicator of cognitive deficits, thus indicating that treatments targeting tau could provide a superior alternative to amyloid beta therapies [45]. Several tau-targeting treatments have been trialled, or are currently being trialled. Most of these focus primarily on kinase inhibition or the inhibition of tau aggregation [48].

These strategies are likely to have a huge benefit for the treatment of $\mathrm{AD}$ and other tauopathies if proven successful but may be limited to treating only patients in the later stages of disease, especially those targeting tau aggregations. Since we find increases in cis p-tau early after TBI in both mouse and human brain, humanisation of our cis p-tau antibody for use as an early therapy could prove to be an effective intervention for patients early after TBI. Furthermore, it could potentially prevent the development of CTE following multiple head traumas. Finally, we have already shown that treatment with our cis p-tau antibody in mouse models not only prevents the development of neurofibrillary tangles and other tau pathologies, it also effectively removes cis p-tau aggregations and improved cognitive function. Therefore, further development and humanisation of the cis p-tau antibody also provides a potential new treatment for prevention and treatment of $\mathrm{AD}$.

\section{Conclusion}

The emergence of cis p-tau as a newly identified pathogenic isoform of tau presents a new pathway through which to develop new diagnostic tests to identify tau pathology following TBI, CTE and to identify early stage
AD. Furthermore, the cis p-tau antibody targets a specific isoform of pathogenic tau which could ultimately be developed into a new treatment method for reducing tau pathology in AD, TBI and CTE and subsequently improving cognitive function in these patients.

\section{Abbreviations \\ AD: Alzheimer's disease; ALS: amyotrophic lateral sclerosis; FTD: frontotempo- ral dementia; NFTs: neurofibrillary tangles; PSP: progressive supranuclear palsy; CBD: corticobasal dementia; CTE: chronic traumatic encephalopathy; TBI: traumatic brain injury; rmTBI: repeated mild traumatic brain injury; $A \beta$ : Amy- loid beta peptide $42 \mathrm{kDa}$; cis p-tau: cis isoform of phosphorylated tau protein; trans-p-tau: trans isoform of phosphorylated tau protein; Pin 1: peptidyl, prolyl isomerase, Pin1.}

\section{Authors' contributions}

$\mathrm{OA}, \mathrm{MKH}$ and SB were responsible for acquisition and interpretation of the literature and drafting and revising the manuscript. All authors gave final approval of the manuscript for publication. XZ and KPL conceived the literature review, $A K, X Z$ and $K P L$ were responsible for revising the manuscript for critically important intellectual content and KPL and XZ agree to be responsible for all aspects of the accuracy and integrity of the work. All authors read and approved the final manuscript.

\section{Acknowledgements \\ Not applicable.}

\section{Competing interests}

Dr. Lu and Dr. Zhou are inventors of Pin 1 technology, which was licensed by BIDMC to Pinteon Therapeutics. Both Dr. Lu and Dr. Zhou own equity in, and consult for, Pinteon and their interests were reviewed and are managed by BIDMC in accordance with its competing interests policy.

\section{Data sharing}

Data sharing not applicable to this article as no datasets were generated or analysed during the current study.

\section{Funding}

OA is an Alzheimer's Association Research Fellow. Work done in the authors' laboratories is supported by NIH grants R01AG029385, R01AG046319, R01CA167677, Alzheimer's Association grant DVT-14-322623, Alzheimer's Association, Alzheimer's Research UK and the Weston Brain Institute grant MCDN-15-368711 to KPL, and gift donations from the Owens Family Foundation to $X Z Z$ and $K P L$

Received: 24 September 2016 Accepted: 24 November 2016

Published online: 05 December 2016

\section{References}

1. Mandelkow EM, Mandelkow E. Biochemistry and cell biology of tau protein in neurofibrillary degeneration. Cold Spring Harbor Perspect Med. 2012;2(7):a006247.

2. Iqbal K, Grundke-Iqbal I. Ubiquitination and abnormal phosphorylation of paired helical filaments in Alzheimer's disease. Mol Neurobiol. 1991;5(2-4):399-410

3. Khatoon S, Grundke-lqbal I, labal K. Levels of normal and abnormally phosphorylated tau in different cellular and regional compartments of Alzheimer disease and control brains. FEBS Lett. 1994;351(1):80-4.

4. Dehmelt L, Halpain S. The MAP2/Tau family of microtubule-associated proteins. Genome Biol. 2004;6(1):1-10.

5. Goedert M, Spillantini MG, Potier MC, Ulrich J, Crowther RA. Cloning and sequencing of the cDNA encoding an isoform of microtubule-associated protein tau containing four tandem repeats: differential expression of tau protein mRNAs in human brain. EMBO J. 1989;8:393. 
6. Buee L, Bussiere T, Buee-Scherrer V, Delacourte A, Hof PR. Tau protein isoforms, phosphorylation and role in neurodegenerative disorders. Brain Res Brain Res Rev. 2000;33(1):95-130.

7. Murray ME, Kouri N, Lin W-L, Jack CR, Dickson DW, Vemuri P. Clinicopathologic assessment and imaging of tauopathies in neurodegenerative dementias. Alzheimers Res Ther. 2014;6(1):1-13.

8. Stoothoff WH, Johnson GVW. Tau phosphorylation: physiological and pathological consequences. Biochim Biophys Acta. 2005;1739(2-3):280-97.

9. Hernandez F, Avila J. Tauopathies. Cellular and molecular life sciences: CMLS. 2007:64(17):2219-33.

10. Johnson VE, Stewart W, Smith DH. Widespread tau and amyloid-beta pathology many years after a single traumatic brain injury in humans. Brain Pathol (Zurich, Switzerland). 2012;22(2):142-9.

11. McKee AC, Stein TD, Kiernan PT, Alvarez VE. The neuropathology of chronic traumatic encephalopathy. Brain Pathol (Zurich, Switzerland). 2015;25(3):350-64.

12. Blennow K, Hardy J, Zetterberg H. The neuropathology and neurobiology of traumatic brain injury. Neuron. 2012;76(5):886-99.

13. Gong CX, Liu F, Grundke-lqbal I, lqbal K. Post-translational modifications of tau protein in Alzheimer's disease. J Neural Transm (Vienna). 2005;112(6):813-38.

14. Martin L, Latypova X, Terro F. Post-translational modifications of tau protein: implications for Alzheimer's disease. Neurochem Int. 2011;58(4):458-71.

15. Min S-W, Chen X, Tracy TE, Li Y, Zhou Y, Wang C, Shirakawa K, Minami SS, Defensor E, Mok SA, et al. Critical role of acetylation in tau-mediated neurodegeneration and cognitive deficits. Nat Med. 2015;21(10):1154-62.

16. Xu L, Zheng J, Margittai M, Nussinov R, Ma B. How does hyperphopsphorylation promote tau aggregation and modulate filament structure and stability? ACS Chem Neurosci. 2016;7(5):565-75.

17. Ballatore C, Lee VMY, Trojanowski JQ. Tau-mediated neurodegeneration in Alzheimer's disease and related disorders. Nat Rev Neurosci. 2007;8(9):663-72.

18. Iqbal K, Liu F, Gong CX. Tau and neurodegenerative disease: the story so far. Nat Rev Neurol. 2016;12(1):15-27.

19. Bilkei-Gorzo A. Genetic mouse models of brain ageing and Alzheimer's disease. Pharmacol Ther. 2014;142(2):244-57.

20. Ojo JO, Mouzon BC, Crawford F. Repetitive head trauma, chronic traumatic encephalopathy and tau: challenges in translating from mice to men. Exp Neurol. 2016;275(Pt 3):389-404.

21. Weingarten MD, Lockwood AH, Hwo SY, Kirschner MW. A protein factor essential for microtubule assembly. Proc Natl Acad Sci USA. 1975;72(5):1858-62.

22. Kanaan NM, Pigino GF, Brady ST, Lazarov O, Binder LI, Morfini GA. Axonal degeneration in Alzheimer's disease: when signaling abnormalities meet the axonal transport system. Exp Neurol. 2013;246:44-53.

23. de Calignon A, Polydoro M, Suarez-Calvet M, William C, Adamowicz DH, Kopeikina KJ, Pitstick R, Sahara N, Ashe KH, Carlson GA, et al. Propagation of tau pathology in a model of early Alzheimer's disease. Neuron. 2012;73(4):685-97.

24. Spires-Jones TL, Stoothoff WH, de Calignon A, Jones PB, Hyman BT. Tau pathophysiology in neurodegeneration: a tangled issue. Trends Neurosci. 2009;32(3):150-9.

25. Hyman BT. Tau propagation, different tau phenotypes, and prion-like properties of tau. Neuron. 2014;82(6):1189-90.

26. Augustinack JC, Schneider A, Mandelkow EM, Hyman BT. Specific tau phosphorylation sites correlate with severity of neuronal cytopathology in Alzheimer's disease. Acta Neuropathol. 2002;103(1):26-35.

27. Biernat J, Gustke N, Drewes G, Mandelkow EM, Mandelkow E. Phosphorylation of Ser262 strongly reduces binding of tau to microtubules: distinction between PHF-like immunoreactivity and microtubule binding. Neuron. 1993;11(1):153-63.

28. Gupta R, Sen N. Traumatic brain injury: a risk factor for neurodegenerative diseases. Rev Neurosci. 2016;27(1):93-100.

29. Stein TD, Alvarez VE, McKee AC. Chronic traumatic encephalopathy: a spectrum of neuropathological changes following repetitive brain trauma in athletes and military personnel. Alzheimers Res Ther. 2014;6(1):4
30. DeKosky ST, Blennow K, Ikonomovic MD, Gandy S. Acute and chronic traumatic encephalopathies: pathogenesis and biomarkers. Nat Rev Neurol. 2013;9(4):192-200.

31. Schmidt ML, Zhukareva V, Newell KL, Lee VM, Trojanowski JQ. Tau isoform profile and phosphorylation state in dementia pugilistica recapitulate Alzheimer's disease. Acta Neuropathol. 2001;101(5):518-24.

32. Mouzon BC, Bachmeier C, Ferro A, Ojo JO, Crynen G, Acker CM, Davies P, Mullan M, Stewart W, Crawford F. Chronic neuropathological and neurobehavioral changes in a repetitive mild traumatic brain injury model. Ann Neurol. 2014;75(2):241-54.

33. Lu PJ, Wulf G, Zhou XZ, Davies P, Lu KP. The prolyl isomerase Pin 1 restores the function of Alzheimer-associated phosphorylated tau protein. Nature. 1999;399(6738):784-8.

34. Yaffe MB, Schutkowski M, Shen M, Zhou XZ, Stukenberg PT, Rahfeld $J U, X u J$ J, Kuang J, Kirschner MW, Fischer G, et al. Sequence-specific and phosphorylation-dependent proline isomerization: a potential mitotic regulatory mechanism. Science (New York, NY). 1997;278(5345):1957-60.

35. Liou YC, Sun A, Ryo A, Zhou XZ, Yu ZX, Huang HK, Uchida T, Bronson R, Bing $G$, Li $X$, et al. Role of the prolyl isomerase Pin1 in protecting against age-dependent neurodegeneration. Nature. 2003;424(6948):556-61.

36. Lim J, Balastik M, Lee TH, Nakamura K, Liou YC, Sun A, Finn G, Pastorino L, Lee VM, Lu KP. Pin 1 has opposite effects on wild-type and P301L tau stability and tauopathy. J Clin Investig. 2008;118(5):1877-89.

37. Nakamura K, Greenwood A, Binder L, Bigio EH, Denial S, Nicholson L, Zhou XZ, Lu KP. Proline isomer-specific antibodies reveal the early pathogenic tau conformation in Alzheimer's disease. Cell. 2012;149(1):232-44.

38. Nakamura K, Zhou XZ, Lu KP. Distinct functions of cis and trans phosphorylated tau in Alzheimer's disease and their therapeutic implications. Curr Mol Med. 2013;13(7):1098-109.

39. Chen CH, Li W, Sultana R, You MH, Kondo A, Shahpasand K, Kim BM, Luo ML, Nechama M, Lin YM, et al. Pin1 cysteine-113 oxidation inhibits its catalytic activity and cellular function in Alzheimer's disease. Neurobiol Dis. 2015;76:13-23.

40. Lee TH, Chen CH, Suizu F, Huang P, Schiene-Fischer C, Daum S, Zhang YJ, Goate A, Chen RH, Zhou XZ, et al. Death-associated protein kinase 1 phosphorylates Pin 1 and inhibits its prolyl isomerase activity and cellular function. Mol Cell. 2011;42(2):147-59.

41. Luna-Munoz J, Chavez-Macias L, Garcia-Sierra F, Mena R. Earliest stages of tau conformational changes are related to the appearance of a sequence of specific phospho-dependent tau epitopes in Alzheimer's disease. J Alzheimers Dis. 2007;12(4):365-75.

42. Lin YT, Cheng JT, Liang LC, Ko CY, Lo YK, Lu PJ. The binding and phosphorylation of Thr231 is critical for Tau's hyperphosphorylation and functional regulation by glycogen synthase kinase 3 beta. J Neurochem. 2007; 103(2):802-13.

43. Kondo A, Shahpasand K, Mannix R, Qiu J, Moncaster J, Chen CH, Yao Y, Lin YM, Driver JA, Sun Y, et al. Antibody against early driver of neurodegeneration cis P-tau blocks brain injury and tauopathy. Nature. 2015;523:431-6.

44. Hawkins BE, Krishnamurthy S, Castillo-Carranza DL, Sengupta U, Prough DS, Jackson GR, DeWitt DS, Kayed R. Rapid accumulation of endogenous tau oligomers in a rat model of traumatic brain injury: possible link between traumatic brain injury and sporadic tauopathies. J Biol Chem. 2013;288(23):17042-50

45. Wisniewski T, Goni F. Immunotherapeutic approaches for Alzheimer's disease. Neuron. 2015:85(6):1162-76.

46. Barthelemy NR, Gabelle A, Hirtz C, Fenaille F, Sergeant N, SchraenMaschke S, Vialaret J, Buee L, Junot C, Becher F, et al. Differential mass spectrometry profiles of tau protein in the cerebrospinal fluid of patients with Alzheimer's disease, progressive supranuclear palsy, and dementia with lewy bodies. J Alzheimers Dis. 2016;51(4):1033-43.

47. Olsson B, Lautner R, Andreasson U, Öhrfelt A, Portelius E, Bjerke M, Hölttä M, Rosén C, Olsson C, Strobel G, et al. CSF and blood biomarkers for the diagnosis of Alzheimer's disease: a systematic review and meta-analysis. Lancet Neurol. 2016;15(7):673-84.

48. Liu YH, Giunta B, Zhou HD, Tan J, Wang YJ. Immunotherapy for Alzheimer disease: the challenge of adverse effects. Nat Rev Neurol. 2012;8(8):465-9. 